\title{
Análise do Modelo CreditRisk+ em uma amostra de portfólio de crédito
}

\author{
Rafael Mileo \\ Mestre em Administração de Empresas pela \\ Universidade Presbiteriana Mackenzie \\ rafaelmileo@msn.com
}

\section{Herbert Kimura}

Doutor em Administração pela Faculdade de Economia, Administração e Contabilidade da Universidade de São Paulo (FEA/USP)

Professor titular da Universidade de Brasília (UnB)

herbert.kimura@gmail.com

\section{Eduardo Kazuo Kayo}

Doutor e Livre-Docente em Administração pela FEA-

USP

Professor do Departamento de Administração (área de

Finanças) da FEA-USP

Coordenador da Divisão de Finanças da ANPAD

(Associação Nacional de Pós-Graduação e Pesquisa em

Administração) e Bolsista de Produtividade em Pesquisa

do $\mathrm{CNPq}$

kayo@usp.br

\section{RESUMO:}

O artigo pretende analisar os fundamentos teóricos e o desempenho do Modelo CreditRisk + , uma das metodologias de gestão de risco de crédito criadas por bancos, em uma amostra de portfólio de crédito. No estudo, o Modelo CreditRisk+ foi aplicado em uma carteira de crédito com financiamentos concedidos entre 1986 e 2009, nos Estados Unidos. Dois procedimentos de análise foram realizados: backtesting, para comparar as medidas de avaliação de risco de perdas de determinado ano aos dados de perda simulados para o ano posterior; teste de estresse, para verificar a sensibilidade do Modelo a mudanças no cenário econômico. Os resultados encontrados sugerem que o Modelo CreditRisk+ subestimou, entre 1997 e 2009, na maioria dos anos analisados, o risco de perdas da amostra de carteira de crédito.

Palavras-chave: Portfólio de Crédito. Risco de Crédito. Gerenciamento de Risco de Crédito. Modelos de Gestão de Portfólio de Crédito. Modelo CreditRisk+.

\section{ABSTRACT}

The paper analyzes CreditRisk+ Model theoretical foundations and fulfillment in a credit portfolio sample. In this analysis, CreditRisk+ Model, one of the risk assessment models created by banks, was applied in an US portfolio sample with default events identified between 1986 e 2009. Two procedures to assess performance were carried out: backtest; to compare losses measures for a given year to loss data simulated for a previous year, and stress test; to verify CreditRisk+ Model sensibility to changes in economic scenario. The results suggest that CreditRisk+ Model underestimated, between 1997 e 2009, in most years studied, the credit risk of the portfolio sample.

Keywords: Credit Portfolio. Credit Risk. Credit Risk Management. Credit Portfolio Management Models. CreditRisk+ Model. 


\section{INTRODUÇÃO}

Historicamente, a quebra de instituições financeiras está associada à ocorrência de inadimplências em massa de financiamentos. Com a interligação dos sistemas financeiros nacionais, os episódios de inadimplementos em massa passaram a ter reflexos em bancos de muitos países. Um exemplo ocorreu em 1990 e 1991, quando os índices de inadimplência atingiram valores próximos a 10\% nos Estados Unidos (CAOUETTE; ALTMAN; NARAYANAN, 1998). Mais recentemente, a crise de 2008 foi outro exemplo de como os problemas de gestão de risco de crédito podem comprometer consideravelmente a segurança do sistema financeiro internacional.

Caouette, Altman e Narayanan (1998) atribuem três causas para a ocorrência de inadimplências em massa no passado: as inadequações na regulamentação; a pressão pela competitividade entre os financiadores; a preocupação excessiva das instituições financeiras no relacionamento com os clientes, que limitou sua experiência na gestão do risco de inadimplência.

Com o objetivo de solucionar o problema das inadequações nas regulamentações mencionado por Caouette, Altman e Narayanan (1998), o Comitê de Supervisão Bancária da Basileia (CSBB) estabeleceu novas diretrizes, em 2004, que têm sido adotadas por diversos países. Os principais objetivos das novas regulamentações internacionais foram: fortalecer a estabilidade do sistema bancário internacional contra crises de fuga de capital e inadimplências em massa e reduzir as diferenças competitivas entre os diferentes países. Para a gestão de risco de crédito, o CSBB estabeleceu a possibilidade de aplicação, pelas instituições financeiras, de modelos internos próprios para o cálculo de capital regulatório (capital econômico mínimo exigido para cobrir os riscos das operações).

De acordo com Patel e Pereira (2007), antes de um modelo de gestão de portfólio ser utilizado formalmente no processo de estabelecimento do capital regulatório para risco de crédito, os reguladores precisam estar confiantes de que o modelo será usado para gerenciar ativamente o risco. Além disso, o modelo precisa ser aprovado conceitualmente, validado empiricamente e produzir requisitos de capital que sejam compatíveis com o nível de risco das instituições.

Os modelos mais antigos de avaliação do risco de crédito tradicionalmente investigavam apenas a concessão em si, ou seja, a decisão de conceder empréstimo para um devedor específico (THOMAS, 2009). Em resumo, qualificavam bons ou maus pagadores, definindo a chance de o empréstimo ficar inadimplente no futuro através de investigações sobre a intenção e a capacidade de pagamento do devedor.

A importância do tema, já que a quebra de bancos muitas vezes está associada à ocorrência de inadimplências em massa, e as novas regulamentações estimularam os bancos e as empresas de consultoria a buscarem novas alternativas de avaliação do risco de crédito, capazes de mensurar o risco do portfólio como um todo. Um dos novos modelos criados pelos bancos foi o CreditRisk+, elaborado pelo CreditSuisse em 1997.

Segundo Gordy (2000), o modelo CreditRisk+ logo assumiu um papel muito importante no mercado financeiro internacional. O modelo transformou-se rapidamente em um influente benchmark para as instituições financeiras. Crouhy, Galai e Mark (2000) corroboram ao afirmar que o modelo CreditRisk+ apresenta a vantagem de ser de relativamente fácil implementação porque não é necessária uma grande quantidade de informações para aplicá-lo.

$\mathrm{O}$ ambiente de novas regulamentações, que estimularam a elaboração de novos modelos de gestão de portfólio de crédito, a necessidade de fortalecer o sistema financeiro internacional contra crises e a importância do modelo CreditRisk + no mercado bancário internacional foram as razões para a realização deste estudo, cujo objetivo é analisar a fundamentação teórica e o desempenho do modelo CreditRisk+ na gestão do risco de crédito, através de sua aplicação em um portfólio de crédito formado por empresas americanas financiadas entre 1986 e 2009.

Tasche (2004) afirma que o modelo CreditRisk+ estima o grau de risco de uma carteira de duas maneiras: através do desvio-padrão da distribuição das perdas ou do dado value-at-risk da distribuição das perdas. Maskara e 
Aggarwal (2009) ponderam que o value-at-risk tornou-se um importante padrão para mensurar eventos extremos e integrar as fontes díspares de risco porque responde a seguinte questão: qual é a perda máxima para um determinado grau de confiança no horizonte de tempo especificado?

O procedimento adotado para analisar o desempenho do modelo CreditRisk+ nesta pesquisa consistiu em uma comparação entre as medidas de perda máxima value-atrisk em diferentes graus de confiança para determinado ano com as perdas médias simuladas para o ano posterior. A análise realizada sugere que o modelo subestima o risco de crédito do portfólio na maioria dos anos estudados.

\section{REFERENCIAL TEÓRICO}

A palavra 'crédito' tem suas origens no termo do latim creditum e significa "ter acreditado" ou "acreditar" (THOMAS, 2009). Em finanças, crédito pode ser definido como a concessão de um recurso financeiro, com a expectativa de recebimento do mesmo valor, mais um prêmio pela concessão, em um determinado espaço de tempo (CAOUETTE; ALTMAN; NARAYANAN, 1998). Ao conceder o recurso, o emprestador acredita que o tomador honrará sua dívida, pagando de volta o total emprestado, acrescido de um valor representativo do custo de oportunidade.

Ao associar o termo do latim creditum ao significado financeiro de crédito, Thomas (2009) afirma que durante 4.000 anos as pessoas acreditaram umas nas outras, emprestando entre si dinheiro ou bens. $\mathrm{O}$ autor menciona um código Sumério, do período de 2.000 a.C., que continha registros de empréstimos em dinheiro feitos por agricultores que deram como contrapartida suas produções agrícolas.

Na década de 1920, Henry Ford, presidente da Ford, e Alfred Pritchard Sloan, então presidente da General Motors, reconheceram não ser suficiente produzir bens de consumo como, por exemplo, carros, para o mercado de massa, se não houvesse meios para financiar a compra desses produtos. Nos últimos 50 anos, finalmente o volume de crédito cresceu, transformando os financiamentos também em um produto das massas
(THOMAS; OLIVER; HAND, 2005).

Caouette, Altman e Narayanan (1998) ponderam que, apesar do risco inerente ao crédito, devido à probabilidade de que o recebimento não se concretize, as operações de financiamento foram um fator fundamental para estimular o desenvolvimento econômico da sociedade europeia.

Maskara e Aggarwal (2009) definem o risco de crédito como o grau de flutuação no valor dos instrumentos de dívidas e derivativos, decorrentes de mudanças na qualidade de crédito do financiado ou contraparte. McQuown (1993) diverge parcialmente dessa definição. Para o autor, o risco de crédito está associado ao default, quando um financiado falha no cumprimento de suas obrigações. A ocorrência de defaults está tipicamente associada a perdas significativas para os financiadores.

Babbel (1989) mencionava o problema do aumento do número de falências bancárias desde 1984. Com o intuito de promover maior segurança ao sistema financeiro internacional, o Comitê de Supervisão Bancária da Basileia publicou, em 1992, o Acordo de Capital da Basileia I, conhecido também como Basileia I, que definiu diferentes graus de riscos para diferentes categorias de ativos (MOHANTY, 2008).

Para Brinkmann e Horvitz (1995), a regulamentação também estabeleceu requisitos de capital para proteger o sistema de segurança de depósitos contra falhas bancárias. Segundo os autores, nos Estados Unidos, desde as décadas de 1970 e 1980, os reguladores já buscavam elevar os montantes de capital regulamentar. Apesar dos esforços para diminuir o risco sistêmico do mercado financeiro, Ojo (2009) argumenta que o Acordo de Capital da Basileia I não foi capaz de eliminar a arbitragem entre capital regulatório e capital econômico. Além disso, segundo a autora, o acordo não fazia distinção entre empréstimos comerciais de diferentes graus de risco, classificando os ativos conforme o tipo e não conforme sua qualidade de crédito.

Em 2004, o Comitê de Supervisão Bancária da Basileia definiu um arcabouço à adequação de capital, incluindo uma nova estrutura para determinar os requisitos mínimos de capital regulamentar ajustado a três tipos 
de risco: crédito, operacional e mercado (MOHANTY, 2008). Além da segmentação em tipos de riscos, as regulamentações do novo Acordo da Basileia, ou Basileia II, enfatizam a flexibilidade para que instituições bancárias adotem seus próprios modelos de classificação para avaliar a qualidade do crédito de seus empréstimos, desde que os modelos sejam aprovados pelo órgão regulador (THOMAS; OLIVER; HAND, 2005).

Nos Estados Unidos, supervisores notaram que as abordagens de mensuração do risco fundamentadas em classificações avançadas próprias das instituições apuravam melhor o risco, sendo viáveis notadamente para as maiores, mais complexas e internacionalmente ativas instituições bancárias (THOMAS, 2009). Especificamente para o caso brasileiro, as instituições de maior porte, com atuação internacional e participação significativa no Sistema Financeiro Nacional (SFN), estão aptas a utilizar a abordagem avançada com base em sistema interno de classificação de risco (BANCO CENTRAL DO BRASIL, 2004).

Thomas, Oliver e Hand (2005) consideram óbvio que os maiores bancos internacionais devem adotar modelos avançados internos de classificação do risco de crédito, porque resultam, em média, em um capital regulamentar requerido $14 \%$ menor para crédito, o que permite ao banco dispor de mais recursos para movimentar e gerar lucros.

A literatura existente sobre risco de crédito permite dividir os tipos de modelos em dois grupos: os modelos de abordagem estrutural e os modelos da forma reduzida (PATEL; PEREIRA, 2007). Para Jarrow (2001), os modelos classificados como estruturais avaliam explicitamente a estrutura de ativos e passivos da empresa financiada. Já os modelos da forma reduzida especificam de maneira exógena uma evolução do spread entre títulos de dívida sem risco e com risco.

Em comum, os dois tipos de modelos têm como propósito estimar a função da distribuição de probabilidades de perdas de um portfólio. Aplicando os modelos em amostras de carteiras, é possível identificar, com base em fundamentos técnicos, carteiras de crédito mais adequadas aos objetivos da instituição financeira e ao grau de risco do portfólio (BRÖKER; SCHWEIZER, 2004).

Especificamente para modelagem de carteiras de crédito, um dos modelos reduzidos mais disseminados é o CreditRisk+ (GORDY, 2000) desenvolvido por Wilde (1997) do CreditSuisse First Boston. O CreditRisk+, no que diz respeito à matemática adotada, é um modelo único entre todos os outros disponíveis para gestão de portfólio de crédito, uma vez que aplica técnicas de matemática atuarial para estimar a distribuição de perdas de um portfólio (GUNDLACH; LEHRBASS, 2004).

Burgisser et al. (1999) consideram que o CreditRisk+ é de fácil implementação prática, graças a algumas características como: (i) baixo número de premissas, (ii) implementação bastante transparente, baseada em conceitos largamente utilizados em seguradoras, (iii) estimação razoavelmente simples da distribuição de perdas através do uso de um procedimento iterativo.

No CreditRisk+, o default é modelado como uma variável aleatória discreta. É possível fazer uma analogia imediata com seguros contra incêndios, por exemplo. Quando um portfólio de casas é segurado, há uma pequena probabilidade de que cada casa seja incendiada, e em geral, a probabilidade de que cada casa queime pode ser vista como um evento independente do incêndio em outras residências (SAUNDERS, 1999).

Assim, cada tipo de empréstimo, como hipotecas e empréstimos para pequenos negócios, pode ser visto da mesma maneira, no que diz respeito ao seu risco de default. Sob o CreditRisk+, cada empréstimo individualmente é visto como detentor de uma pequena probabilidade de default, e as probabilidades de default dos empréstimos são independentes entre si.

Crouhy, Galai e Mark (2000) afirmam que o foco do modelo CreditRisk + original é a inadimplência. O CreditRisk + assume que a frequência de inadimplências de empréstimos aproxima-se a uma distribuição Poisson. A migração do risco de crédito não é explicitamente modelada nessa análise. O CreditRisk + permite taxas de default estocásticas com contribuição parcial para o risco de migração. O principal direcionador do risco no CreditRisk+ é o índice médio de inadimplência 
da economia. Esse índice pode ser visto como sistematicamente conectado ao estado da macroeconomia. Quando a macroeconomia deteriora-se, a taxa de default média tende a crescer, assim como as perdas em defaults. Uma melhora das condições econômicas tem o efeito oposto (SAUNDERS, 1999).

Gundlach e Lehrbass (2004) acrescentam que o empréstimo, no CreditRisk+, assume a condição de uma variável aleatória de Bernoulli. Há apenas duas situações possíveis: ou o devedor paga o valor financiado, ou ocorre um evento de inadimplência. Cada financiamento do portfólio não tem a mesma distribuição Bernoulli porque a probabilidade de default de cada ativo pode variar. Segundo Crouhy, Galai e Mark (2000), no CreditRisk+ não são feitos questionamentos sobre as causas do default: um financiado $A$ entrará em default com a probabilidade $P_{A}$ ou não entrará em default coma probabilidade $1-p_{A}$.

Capuano et al. (2009) atribuem a irrelevância das causas do default ao fato de o CreditRisk + ser um modelo da forma reduzida. Contrariamente aos modelos estruturais, os modelos da forma reduzida consideram que os eventos de default não dependem das características da empresa financiada. Segundo Çetin et al. (2004), modelos da forma reduzida transformaram-se em uma ferramenta importante para o gerenciamento do risco de crédito porque usualmente adequam-se melhor aos dados de mercado do que modelos estruturais.

Conforme Gundlach (2004), podem ser elencadas como características fundamentais do CreditRisk+: (i) desinteresse pelas causas do default; o default é um evento puramente aleatório, marcado por uma probabilidade de ocorrência, (ii) probabilidade de default estocástica; a probabilidade de default de um devedor não é vista como uma constante, mas como um valor que varia aleatoriamente, direcionado por um ou mais fatores de riscos (sistemáticos e específicos), (iii) dependência linear dos fatores de risco; há uma relação de dependência linear entre os fatores de riscos sistemáticos e as probabilidades de default assumidas, (iv) independência condicional; dados os fatores de risco, os defaults de devedores são independentes entre si, (v) correlações implícitas entre os direcionadores de risco; as correlações entre os devedores não são explícitas, mas originam-se implicitamente de fatores de risco comuns capazes de direcionar a probabilidade de defaults, (vi) perdas discretas; para agregar as perdas de um portfólio de forma confortável, as perdas são representadas como múltiplos de uma unidade de perda.

Para Reib (2004), a premissa da independência condicional é muito rigorosa do ponto de vista prático. Assim, versões menos restritivas do modelo podem introduzir setores para classes gerais de negócios. Os setores podem estar correlacionados e serem dependentes, através de uma matriz de correlação. Para estimar essas correlações, podem ser utilizadas correlações aproximadas entre os índices dos ativos das empresas.

De modo semelhante, Saunders, Costa e Zenios (2007) afirmam que os eventos de inadimplência dos financiados não são independentes entre si. A correlação entre os defaults decorre do fato de que os financiados podem estar no mesmo segmento ou em segmentos semelhantes da economia, assim como podem estar suscetíveis aos mesmos fatores macroeconômicos.

Boegelein et al. (2004) complementam a questão de dependência ou independência exemplificando que cada devedor de um portfólio de crédito pode ser alocado em uma série de setores, com fatores de risco independentes representados por uma variável aleatória contínua de distribuição conhecida. As frequências de default de cada devedor de um mesmo setor são dependentes em um fator aleatório comum e permitem uma modelagem de eventos de default correlacionados.

Burgisser et al. (1999) aplicam modificações no modelo padrão do CreditRisk + , atribuindo fatores de correlações entre os setores da indústria. Segundo os autores, as correlações podem ser adicionadas de três maneiras: (i) usando séries temporais históricas de eventos de default observados nas indústrias; (ii) usando valores dos ativos das empresas para derivar correlações entre pares de ativos, que depois podem ser associadas a correlações entre defaults utilizando o modelo de Merton (1974); (iii) usando um modelo fatorial que relacione o número de defaults a fatores macroeconômicos. 
A taxa de default é apenas um tipo de incerteza modelada no CreditRisk+. A segunda incerteza envolve o tamanho da perda, isto é, a severidade das perdas. Para entender o conceito de severidade das perdas pode ser utilizada novamente a analogia do incêndio de imóveis. Quando uma casa pega fogo, o grau de severidade da perda pode variar entre avarias no telhado ou a completa destruição do imóvel, por exemplo. No CreditRisk+, sabe-se que as taxas de severidade são incertas, mas, por causa da dificuldade em medir a severidade de cada default individualmente, as exposições de crédito são agrupadas (GORDY, 2000).

Assim, os dados de entrada chave são taxas de perdas principais e severidade das perdas para vários grupos de portfólios de crédito. Cada grupo de empréstimos pode ser entendido como um portfólio separado, e a distribuição das perdas totais é o agrupamento das distribuições de perdas individuais (SAUNDERS, 1999). Para Tasche (2004), há duas maneiras de realizar o cálculo do capital requerido no CreditRisk+: (i) desvio-padrão da distribuição das perdas, ou (ii) value-at-risk (VaR) da distribuição das perdas, que indica o valor de uma perda para um dado percentil.

\section{PROCEDIMENTOS METODOLÓGICOS}

O Modelo CreditRisk+ foi aplicado em uma carteira de crédito formada por empresas norte-americanas com financiamentos obtidos entre 1986 e 2009 (dados retirados da plataforma Bloomberg). A amostra de portfólio continha registros de eventos de inadimplência das empresas financiadas ocorridos no período.

A técnica utilizada para chegar à distribuição de perdas médias e VaRs, resultados finais para a gestão do risco de crédito com o Modelo CreditRisk+, foi gerar duas distribuições: (i) uma para a frequência dos eventos de default e (ii) outra para a severidade das perdas ocasionadas pelos defaults. De acordo com Melchiori (2004), a metodologia da frequência/severidade é uma das mais flexíveis para avaliar as perdas originadas em portfólios de seguradoras. Em modelos de crédito, as médias de frequência e severidade de perdas são utilizadas para calcular a perda esperada em função de potenciais inadimplências.
Ainda segundo Melchiori (2004), a frequência representa o número de eventos de default de um portfólio em um determinado período de tempo. O número médio esperado de eventos de inadimplência, $X$, somente pode assumir, portanto, o valor de números inteiros não negativos, isto é, a frequência de inadimplências é uma variável aleatória discreta representativa do número de inadimplências de uma carteira.

O modelo CreditRisk+ assume que há um grande número de exposições a perdas em uma carteira de crédito e que a probabilidade de que cada financiado em particular entre em situação de default é pequena. Nessas circunstâncias, a distribuição do número de eventos de inadimplência de um portfólio será muito próxima a uma distribuição de Poisson (WILDE, 1997).

A distribuição de Poisson necessita de apenas um parâmetro, o número esperado de eventos de inadimplência ${ }^{\mu}$. A estimativa do parâmetro ${ }^{\mu}$ foi obtida pelo cálculo da média aritmética dos dados de default ocorridos no período estudado.

Neste artigo, para uma janela de tempo com títulos emitidos entre 1986 e 1995, verificou-se a quantidade de títulos inadimplentes no ano seguinte ao último ano da janela, nesse caso, 1996. Para o período entre 1987 e 1996, identificou-se o número de eventos de inadimplência em 1997, e assim sucessivamente. Dessa forma, foram utilizados dados passados de 10 anos para estimar o número esperado de defaults para o próximo período, conforme a Tabela 1. Como a plataforma Bloomberg fornecia o número de eventos de inadimplência ano após ano, foi calculado o índice médio de inadimplências da amostra através de janelas de tempo de dez anos.

A média aritmética de eventos de inadimplência na análise de janelas móveis foi de 48,3571, isto é, o parâmetro ${ }^{\mu}$ inicial de entrada utilizado na distribuição de Poisson para gerar os dados de frequências de default esperados para todo o período foi 48 , conforme os dados apresentados na Tabela 1.apresentados na tabela 1. 


\begin{tabular}{ccc}
\hline \multicolumn{3}{c}{ Tabela 1 - Janelas móveis e defaults } \\
\hline Janela Móvel & Defaults no ano seguinte & $\begin{array}{c}\text { Títulos vencidos a } \\
\text { partir do ano } \\
\text { seguinte }\end{array}$ \\
\hline $86-95$ & & 25588 \\
$87-96$ & 6 & 26308 \\
$88-97$ & 13 & 26574 \\
$89-98$ & 38 & 26207 \\
$90-99$ & 55 & 25302 \\
$91-00$ & 160 & 23002 \\
$92-01$ & 148 & 21577 \\
$93-02$ & 31 & 19082 \\
$94-03$ & 10 & 16665 \\
$95-04$ & 66 & 15593 \\
$96-05$ & 19 & 14979 \\
$97-06$ & 7 & 14361 \\
$98-07$ & 20 & 13999 \\
$99-08$ & 100 & 13776 \\
\hline Médias & 48.3571 & 20215.2143 \\
\hline
\end{tabular}

Fonte: os autores com base em dados da Bloomberg

$\mathrm{Na}$ modelagem da severidade das perdas, foi utilizado como base um estudo de Verde et al. (2009), que classificava o portfólio de instituições inadimplentes em faixas de recuperações das inadimplências, entre $2000 \mathrm{e}$ 2008, nos Estados Unidos. Em seu agrupamento, Verde et al. (2009) estabeleceram seis diferentes faixas de recuperações do defaults, para cada cem empresas em situação de default, conforme a Tabela 2.

Tabela 2 - Classificação de empresas em faixas de recuperações

\begin{tabular}{cc}
\hline Faixa de Recuperação (\%) & $\begin{array}{c}\text { Empresas inadimplentes na } \\
\text { faixa (a cada 100) }\end{array}$ \\
$0>10$ & 5 \\
$10>30$ & 20 \\
$30>50$ & 27 \\
$50>70$ & 23 \\
$70>90$ & 14 \\
$>90$ & 11
\end{tabular}

Fonte: Verde et al. (2009)

Segundo o agrupamento de Verde et al. (2009), de cada cem financiamentos não pagos entre 2000 e 2008, os bancos recuperaram: entre 0 e $10 \%$ do valor em 5 casos; entre $10 \%$ e $30 \%$ em 20 casos; entre $30 \%$ e $50 \%$ em 27 casos; entre $50 \%$ e $70 \%$ em 23 casos; entre $70 \%$ e
90\% em 14 casos; mais do que $90 \%$ do valor de face do financiamento em 11 casos.

Neste artigo, foi utilizado o método estatístico relatado por Stevenson (1981), para calcular, de forma aproximada, a média, a variância e o desvio-padrão dos dados agrupados.

Em primeiro lugar, foi calculada a média de recuperações de cada faixa de recuperação, ${ }^{x_{i}}$. Em seguida, multiplicouse a frequência de eventos de recuperação de dada faixa, $f_{i}$, pela média de recuperações do mesmo grupo. O procedimento foi repetido para todos os grupos, somando os resultados. O resultado final foi dividido pelo total de eventos de inadimplência, ${ }^{n}$, que no caso do estudo de Verde et al. (2009), foi de cem, para obter a média de recuperação de todo o agrupamento.

$$
\bar{x}=\frac{f_{1} x_{1}+f_{2} x_{2}+\cdots+f_{n} x_{n}}{n}
$$

Em seu estudo, Stevenson (1981) realiza o cálculo da variância de dados grupados conforme a equação abaixo:

$$
\text { Variância }=\frac{\sum f\left(x_{i}-x\right)^{2}}{n-1}
$$

O cálculo da variância, portanto, foi realizado pelo somatório das multiplicações das frequências pelos erros quadrados de cada grupo em relação à recuperação média, $\bar{x}$, de todo o agrupamento. Ao final, foi dividido o somatório pelo total de frequências menos o número um. O desvio-padrão foi calculado pela raiz quadrada da variância. Os resultados dos cálculos realizados estão demonstrados na Tabela 3 abaixo.

\begin{tabular}{|c|c|c|c|c|c|c|c|}
\hline \multicolumn{8}{|c|}{ severidades (dados por grupo). } \\
\hline Grupos & $\begin{array}{c}\text { Média } \\
\text { dos } \\
\text { grupos } \\
\left(x_{i}\right)\end{array}$ & $\bar{x}$ & $\begin{array}{l}\text { Frequência } \\
\qquad\left(f_{i}\right)\end{array}$ & $f_{i} \times x_{i}$ & $\left(x_{i}-\bar{x}\right)^{2}$ & $f_{i}\left(x_{i}-\bar{x}\right)^{2}$ & $\begin{array}{l}\text { Média de } \\
\text { perdas }\end{array}$ \\
\hline $0>10$ & 0,05 & 0,505 & 5 & 0,25 & 0,207025 & 1,035125 & 0,505 \\
\hline $10>30$ & 0,2 & 0,505 & 20 & 4 & 0,093025 & 1,8605 & \\
\hline $30>50$ & 0,4 & 0,505 & 27 & 10,8 & 0,011025 & 0,297675 & Variância \\
\hline $50>70$ & 0,6 & 0,505 & 23 & 13,8 & 0,009025 & 0,207575 & 0,068661616 \\
\hline $70>90$ & 0,8 & 0,505 & 14 & 11,2 & 0,087025 & 1,21835 & \\
\hline$>90$ & 0,95 & 0,505 & 11 & 10,45 & 0,198025 & 2,178275 & $\begin{array}{l}\text { Desvio- } \\
\text { padrão }\end{array}$ \\
\hline & & & 100 & 50,5 & & 6,7975 & 0,262033616 \\
\hline
\end{tabular}

Tabela 3 - Média, variância e desvio padrão das

Fonte: Verde et al. (2009, p. 11). 
Segundo Melchiori (2004), para gerar as distribuições finais de perdas acumuladas da carteira de crédito, o CreditRisk + considera as severidades das perdas como variáveis aleatória contínuas.

A média das perdas, 50,5\%, e o desvio-padrão das perdas, $26,20 \%$, calculados com base no estudo de Verde et al. (2009) para os dados agrupados, foram utilizados para deduzir os parâmetros das distribuições lognormal e gamma, distribuições adequadas para modelar variáveis aleatórias contínuas, como a severidade de perdas.

Após a obtenção dos parâmetros da distribuição de Poisson, para modelar a frequência de eventos de inadimplência, e das distribuições lognormal e gamma, para modelar as severidades das perdas, foram desenvolvidas subrotinas em macros de Excel com o objetivo de integrar a distribuição de frequência às distribuições de severidades. Na sub-rotina, a quantidade de perdas era simulada e, para cada perda, gerada aleatoriamente uma severidade.

A simulação foi realizada, portanto, sorteando um valor $N$ para o número de defaults através de distribuições de frequência de Poisson. Para cada default, era gerado ao acaso um valor de perda com a distribuição de severidade, $X_{i}$, com $0 \leq i \leq N$. Ao final, as perdas geradas para cada default eram acumuladas, obtendo a perda total no período, $S$ :

$$
S=\sum_{i=1}^{N} X_{i}
$$

As sub-rotinas criadas em macro do Excel acumulavam as perdas geradas, integrando a modelagem da frequência e das severidades dos eventos de inadimplência. Em resumo, eram simuladas quantidades de eventos de inadimplência e, para cada evento, um determinado valor de perda não recuperado. Ao final, as severidades dos eventos eram acumuladas para obter um valor de perda total da carteira.

Cada procedimento reproduzido pela sub-rotina criava automaticamente cinco mil resultados de perdas acumuladas. A cada cinco mil perdas acumuladas, a subrotina gerava três dados de value-at-risk, um para cada grau de confiança $(90 \%, 95 \%$ e 99\%), e um dado de perda média da carteira para cada ano. O mecanismo de geração de value-at-risk e perda efetiva para a carteira foi repetido duzentas vezes em cada um dos três graus de confiança.

Os duzentos resultados de perdas efetivas da carteira e dados value-at-risk (em seus três graus de confiança) obtidos pela sub-rotina foram utilizados para calcular a média anual de perdas da carteira e os dados VaR de perda máxima anual esperada em seus três graus de confiança.

Em seguida, foram aplicadas alterações: (i) no parâmetro $\mu$ da distribuição de Poisson, responsável por modelar a frequência de eventos, (ii) na esperança e no desviopadrão necessários para os cálculos dos parâmetros das distribuições lognormal e gamma, responsáveis pela modelagem das severidades. As alterações aplicadas nos parâmetros poderiam simular melhores ou piores condições no mercado de crédito. Um aumento no parâmetro $\mu$ da distribuição de Poisson, por exemplo, poderia significar um crescimento no número de eventos de inadimplência ocasionado por um cenário de crise na macroeconomia. Uma redução do número de inadimplências, ao contrário, poderia ser efeito de uma melhora nas condições econômicas. As alterações aplicadas aos parâmetros das distribuições permitiram, em resumo, analisar o comportamento do modelo em situações de mudanças relevantes de cenário de crédito. O objetivo principal do procedimento de análise de sensibilidade a alterações nos parâmetros foi verificar os resultados obtidos pelo modelo CreditRisk+ em um cenário de estresse no qual a quantidade de eventos ou a severidade das perdas aumentassem consideravelmente.

Nesta pesquisa, inicialmente foi alterada a frequência de eventos de inadimplências no período estudado. $\mathrm{O}$ número de inadimplências médio inicial era de 48 eventos para uma média de 20.215 títulos de dívida válidos. A quantidade média de eventos de inadimplência foi alterada para valores entre cinco e quinhentos eventos, mantendo o número médio de títulos válidos inalterado.

$\mathrm{Na}$ severidade das perdas, a esperança inicial utilizada para gerar os parâmetros das distribuições lognormal e gamma foi de $50,50 \%$, o que significava que, a cada 
inadimplência, o banco perdia em média 50,50\% do valor total do financiamento. O desvio-padrão inicial utilizado para calcular os parâmetros das duas distribuições foi $26,20 \%$. Se alterada a esperança de $50,50 \%$ para $10 \%$, significaria que na média os financiamentos passaram a perder apenas $10 \%$ do seu valor total.

Foram aplicadas, então, alterações de 10 em 10 pontos percentuais, entre $10 \%$ e $100 \%$, na esperança das distribuições lognomal e gamma. Quando utilizada a distribuição gamma, no entanto, com o valor da esperança em $10 \%$ e $20 \%$, não foi possível modelar o fenômeno severidade da maneira adequada. A distribuição gamma depende de dois parâmetros: escala $(\beta)$ e forma $(\alpha)$. Com a esperança em $10 \%$ ou $20 \%$, o desvio-padrão ficou maior do que a esperança, o que deixou o parâmetro forma da distribuição em um valor menor do que 1, impossibilitando a sua utilização. Foi necessário alterar também o desviopadrão, na distribuição gamma, para um valor abaixo de $10 \%$, com o objetivo de manter o parâmetro forma da distribuição sempre maior do que 1 . O valor do desviopadrão atribuído foi de 0,09 , permitindo assim modelar a severidade também com a distribuição gamma.

Ao final, para analisar o desempenho inicial (isto é, sem alterações nos parâmetros) do modelo CreditRisk+ na mensuração dos dados de perdas efetivas acumuladas na carteira no período entre 1997 e 2009, foi realizado o procedimento de backtesting. O backtesting consistiu em um comparativo entre os VaRs, obtidos com os dados do ano 2000, por exemplo, e as perdas efetivas da carteira no ano imediatamente posterior, 2001, no caso acima.

Se os values-at-risk de perda máxima para o ano 2000, por exemplo, fossem maiores do que as perdas efetivas de 2001, a medida de risco de crédito gerada pelo CreditRisk+ estaria em conformidade ao risco da carteira. Se as perdas de crédito do ano 2001 superassem os valuesat-risk estimados para o ano 2000, haveria, ao contrário, uma não conformidade do modelo na mensuração do risco de crédito. O procedimento de backtesting supõe que a carteira dos dois anos comparados é homogênea, no sentido de ser composta por títulos semelhantes.

É importante ressaltar que as não conformidades não indicam necessariamente um resultado ruim, uma vez que o value-at-risk é estimado com um grau de confiança. Assim, por exemplo, para um VaR obtido com 90\% de confiança, é de se esperar que, em termos médios, a perda efetiva supere o VaR em $10 \%$ dos anos, ou seja, uma vez a cada 10 anos.

\section{ANÁLISE DE RESULTADOS}

A partir dos dados de perdas efetivas gerados pela subrotina para cada um dos 13 anos estudados, entre 1997 e 2009, foi calculada a perda média da carteira analisada em todo o período. Os resultados indicam que, durante os 13 anos analisados, a perda média anual, tanto com a distribuição lognormal, quanto com a distribuição gamma, foi de $0,121 \%$ do valor total financiado. Para a estimação das perdas médias da carteira, os resultados sugerem que utilizar a distribuição de severidade lognormal ou gamma não é relevante para a modelagem do risco de crédito com o CreditRisk + .

Tabela 4 - Perdas médias em função de diferentes

\begin{tabular}{cc}
\multicolumn{2}{c}{ distribuições de severidade } \\
\hline \multicolumn{2}{c}{ Perdas médias } \\
\hline Lognormal & Gamma \\
$0.121 \%$ & $0.121 \%$ \\
\hline
\end{tabular}

Fonte: os autores

Os dados value-at-risk gerados pela sub-rotina para os 13 anos estudados também foram obtidos, em seus três graus de confiança, 90\%, 95\% e 99\%, com as duas distribuições de severidade: lognormal e gamma. Os valores de perdas máximas potenciais anuais, VaRs, gerados com as duas distribuições de severidade (lognormal e gamma) foram muito próximos. Assim como o observado nas perdas médias, na geração dos dados value-at-risk a utilização da distribuição de severidade lognormal ou gamma mostrou-se indiferente para a modelagem do risco de crédito do portfólio com o CreditRisk+.

Para cada ano, a sub-rotina gerava 200 dados VaR, que medem a perda máxima com $90 \%, 95 \%$ e $99 \%$ de confiança. Em seguida, era feito o cálculo da média aritmética anual dos duzentos dados VaR gerados nos três graus de confiança. Com a média aritmética das médias anuais, foi calculada a média de perda máxima anual para todo o período. Com base nos dados entre 1997 e 2009, 
os resultados obtidos de perda média máxima anual, portanto, foram: $0,146 \%$, a $90 \%$ de confiança; $0,153 \%$, a $95 \%$ de confiança; e $0,167 \%$ a $99 \%$ de confiança. Os resultados indicaram que a chance de a perda da carteira em um ano ser superior a $0,146 \%$ seria de no máximo $10 \%$. A probabilidade de ser superior a $0,153 \%$ seria de no máximo $5 \%$, e, finalmente, o risco de uma perda maior do que $0,167 \%$ seria de no máximo $1 \%$.

Uma análise interessante realizada após a obtenção das perdas efetivas anuais foi identificar a perda mínima anual e a perda máxima anual no período estudado. Nessa análise, foram geradas frequências de eventos de inadimplência usando como referência os anos com a menor e com a maior quantidade de eventos de inadimplência, simulando, para cada um dos dois anos, distribuições de severidade. Os anos utilizados foram: 1996, com apenas quatro defaults; e 2001, com 160 eventos de inadimplência.

Entre 1996 e 2009, a menor perda média anual foi de aproximadamente $0,008 \%$, em 1996, enquanto a maior perda média anual foi de $0,351 \%$ do valor total financiado, em 2001. Na prática, isso significa que, em portfólios semelhantes aos estudados, a maioria das perdas ficaria entre esses dois valores mencionados.

Tabela 5 - Estimativas de perdas para frequência máxima e mínima do período

\begin{tabular}{cc}
\hline \multicolumn{2}{c}{ Lognormal } \\
\hline 1996 & 2001 \\
$0,008 \%$ & $0,351 \%$ \\
\hline \multicolumn{2}{c}{ Gamma } \\
\hline 1996 & 2001 \\
$0,008 \%$ & $0,351 \%$
\end{tabular}

Fonte: os autores

Os resultados obtidos indicam que o parâmetro ${ }^{\mu}$ da distribuição de Poisson, isto é, a quantidade média de eventos de inadimplência, é muito importante para o resultado de perdas efetivas do portfólio de crédito. A alta volatilidade percebida na quantidade de eventos de inadimplência do portfólio entre 1997 e 2009 fez com que a diferença entre as perdas médias efetivas obtidas entre o ano de melhor e o ano de pior cenário fosse muito elevada. A grande diferença verificada indica que testes em cenários extremos, especialmente em relação à frequência de defaults, são importantes para complementar a análise do modelo CreditRisk+.

A primeira análise de comportamento em face de mudanças de cenário consistiu em alterações no parâmetro $\mu$ da distribuição de Poisson. O parâmetro $\mu$ inicial, de 48 eventos, foi alterado para valores entre cinco (melhora do cenário no mercado de crédito) e quinhentos (piora do cenário para crédito). Os parâmetros das distribuições de severidade foram mantidos, o que significa que para cada evento era gerada uma perda constante de 50,5\% do valor total do financiamento inadimplente. A quantidade média de financiamentos da carteira, 20.215, também não foi alterada.

O efeito das alterações no parâmetro da distribuição de frequência não trouxe resultados surpreendentes. Ao passo que o número de eventos de inadimplência aumentava, os VaRs (em todos os seus graus de confiança) e as perdas médias elevaram-se gradativamente. Com 500 eventos de inadimplência na carteira de, em média, 20.215 concessões, a perda média do portfólio ficou próxima de $1,2 \%$ do total financiado.

Figura 1 - Perdas e VaR após alterações no parâmetro $\mu$ da distribuição de Poisson

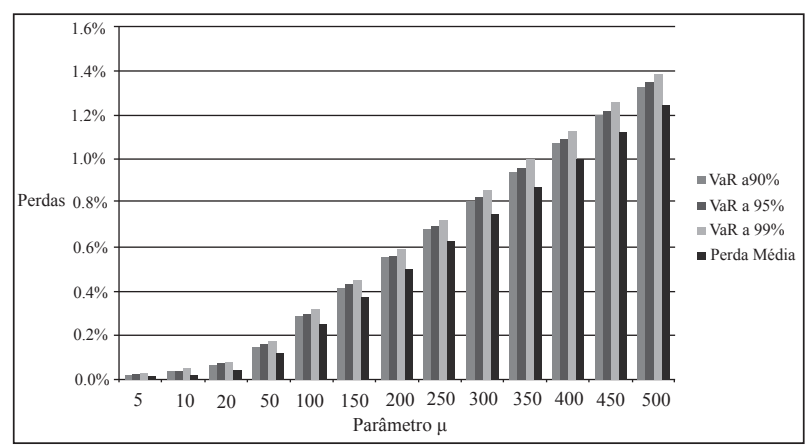

Fonte: os autores

Para a análise do modelo CreditRisk + ante alterações nas severidades das perdas, foi alterada, inicialmente, a esperança utilizada para o cálculo dos parâmetros das distribuições lognormal e gamma. A esperança foi alterada para valores entre $10 \%$ e $100 \%$. Até o momento em que a esperança estivesse em 50\%, estariam sendo simuladas condições melhores no mercado de crédito, com perdas menos severas, uma vez que as simulações iniciais foram realizadas com perdas médias de 50,50\% do valor financiado em cada evento de inadimplência. 
A partir de uma esperança a $60 \%$, o cenário de crédito apresentaria condições piores do que a realidade inicialmente simulada. O desvio-padrão, 26,20\%, a quantidade média de financiamentos em carteira, 20.215, e o número médio de eventos de inadimplência, 48, não foram alterados nas simulações. O objetivo foi verificar os resultados obtidos com o CreditRisk + diante apenas de mudanças na severidade das perdas.

Com a severidade das perdas em $100 \%$ para cada evento de inadimplência, a perda média anual da carteira de crédito no período entre 1997 e 2009 atingiu um patamar próximo a $0,25 \%$ do total financiado.

Figura 2 - Perdas após alterações na esperança com a distribuição gamma

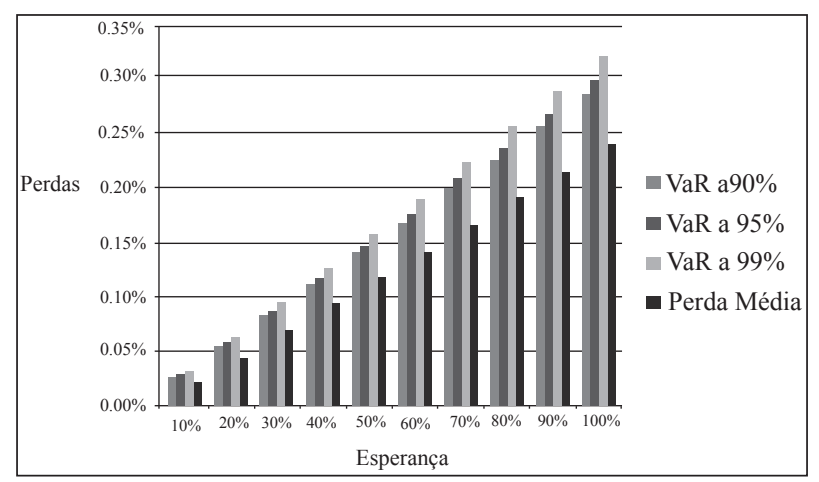

Fonte: os autores

O gráfico de comportamento das perdas após as alterações na esperança das severidades, quando utilizada a distribuição lognormal, não é apresentado porque os resultados foram muito semelhantes aos obtidos com a distribuição gamma.

Posteriormente, foram aplicadas alterações também na variância utilizada para o cálculo dos parâmetros das distribuições lognormal e gamma. Quando modificados, os valores da variância provocavam alterações no desviopadrão, dado pela sua raiz quadrada. As variações realizadas na variância (e consequentemente no desviopadrão) do fenômeno severidade não geraram efeitos tão rigorosos quanto as variações efetuadas na esperança das severidades.

Com o objetivo de analisar o desempenho do modelo CreditRisk+, a Tabela 5 abaixo apresenta um comparativo das medidas de risco de crédito obtidas sem alterações nos parâmetros das distribuições, VaRs, com as perdas efetivas identificadas pelas simulações ano a ano no período entre 1997 e 2009.

Embora os valores de VaR devessem aumentar com o aumento do grau de confiança, em alguns anos esse fato não ocorreu porque, apesar de seguir distribuições de frequência e severidades preestabelecidas, o processo de produção de observações de variáveis aleatórias partir de uma distribuição, isto é, o processo de geração de realizações de variáveis aleatória pode gerar simulações com valores bem superiores à média.

Tabela 6 - Resultados do backtesting do CreditRisk+

\begin{tabular}{ccccccccc}
\hline & $\begin{array}{c}\text { Perda } \\
\text { efetiva }\end{array}$ & Ano (eventos) & $\mathbf{9 0} \%$ & $\mathbf{9 5 \%}$ & $\mathbf{9 9 \%}$ & $\mathbf{9 0 \%}$ & $\mathbf{9 5 \%}$ & $\mathbf{9 9 \%}$ \\
\hline 1997 & 0.000115174 & $1996(4)-1997(6)$ & 0.000022 & 0.000043 & 0.000083 & $2.3 \mathrm{E}-05$ & 0.000044 & 0.000085 \\
1998 & 0.000247046 & $1997(6)-1998(13)$ & 0.000062 & 0.00004 & 0.000059 & 0.00006 & 0.000039 & 0.000038 \\
1999 & 0.000732247 & $1998(13)-1999(38)$ & 0.00039 & 0.0004 & 0.0003 & 0.0004 & 0.00035 & 0.0003 \\
2000 & 0.001097739 & $1999(38)-2000(55)$ & 0.00019 & 0.0001 & 0.00005 & 0.0002 & 0.00014 & 0.00005 \\
2001 & 0.003512738 & $2000(55)-2001(160)$ & 0.0022 & 0.0021 & 0.002 & 0.0022 & 0.00213 & 0.002 \\
2002 & 0.003463874 & $2001(160)-2002(148)$ & 0.000445 & 0.00056 & 0.00076 & 0.00044 & 0.00056 & 0.00077 \\
2003 & 0.000820407 & $2002(148)-2003(31)$ & 0.003054 & 0.00317 & 0.00339 & 0.00306 & 0.003176 & 0.00339 \\
2004 & 0.00030303 & $2003(31)-2004(10)$ & 0.000734 & 0.0008 & 0.00092 & 0.00073 & 0.000798 & 0.00091 \\
2005 & 0.002137498 & $2004(10)-2005(66)$ & 0.00169 & 0.0016 & 0.0016 & 0.0017 & 0.00165 & 0.0016 \\
2006 & 0.000640563 & $2005(66)-2006(19)$ & 0.001879 & 0.00199 & 0.00219 & 0.00188 & 0.00199 & 0.00219 \\
2007 & 0.000246153 & $2006(19)-2007(7)$ & 0.000609 & 0.00068 & 0.0008 & 0.00061 & 0.000679 & 0.0008 \\
2008 & 0.00072148 & $2007(7)-2008(20)$ & 0.00034 & 0.0003 & 0.0002 & 0.0003 & 0.00029 & 0.0002 \\
2009 & 0.003665796 & $2008(20)-2009(100)$ & 0.00271 & 0.0026 & 0.0025 & 0.0027 & 0.00264 & 0.0025 \\
\hline
\end{tabular}

Fonte: os autores

A Tabela 6 deve ser interpretada da seguinte maneira: com base no parâmetro $\mu$ de seis eventos de inadimplência identificados em 1996 e no dado de severidade de $50,5 \%$ de perda em cada evento, a perda média efetiva da carteira foi de 0,000115174, em 1997. A média dos 200 dados VaR obtidos para 1996 a 90\% de confiança foi de 0,000022. O questionamento que o backtesting tenta esclarecer é: a média das estimativas VaR a $90 \%$ de confiança obtida para 1996 foi uma boa medida para o risco de crédito de 1997 ?

No backtesting, o mesmo procedimento de comparação foi realizado para todos os anos estudados. Os dados VaR, estimados em três graus de confiança, de um determinado ano, $X$, foram comparados com as perdas médias efetivas do ano posterior, $X+1$. Ao analisar de maneira comparativa os dados VaR com as perdas médias efetivas simuladas, uma vez que em cada dez comparações poderia ocorrer uma perda média efetiva maior em $X+$ 1 do que o VaR obtido em $X$, na estimativa com $90 \%$ de confiança.

As perdas máximas potenciais mensuradas através de 
values-at-risk para o ano 2000, por exemplo, indicavam que, com $90 \%$ de confiança, as perdas não ultrapassariam, em 2001, o valor de 0,00019. Em 2001, quando o número de eventos de inadimplência foi muito maior, 160 contra 55 do ano 2000, a perda média efetiva foi de 0,0035 , bem maior do que os 0,00019 estimados pelo VaR com 90\% de confiança no ano anterior. A mesma não conformidade identificada no ano 2000 foi percebida também em outras nove das treze comparações realizadas no backtesting

\section{CONCLUSÕES}

Em 1997, 1998, 1999, 2000, 2001, 2002, 2005, 2008 e 2009, isto é, em nove dos treze anos analisados, as estimativas value-at-risk a $90 \%$ de confiança, feitas para o ano imediatamente anterior, não foram medidas adequadas para mensurar o risco de crédito. As nove não conformidades nas comparações entre as estimativas VaR a $90 \%$ de confiança para determinado ano $X$ e as perdas médias efetivas anuais identificadas no ano $X+1$ indicam que as estimativas value-at-risk não representaram, na amostra estudada, boas medidas de risco de crédito para os anos subsequentes. O procedimento de comparação ano a ano realizado indica que as estimativas de value-at-risk, a $90 \%, 95 \%$ ou $99 \%$ de confiança, ou seja, as medidas de risco geradas pelo CreditRisk+ subestimaram, de uma forma geral, o risco de crédito da carteira. Na amostra estudada, portanto, o CreditRisk + indicou um nível de risco de crédito inferior ao efetivo.

Várias são as razões possíveis para a não conformidade do modelo na análise de risco de crédito da carteira estudada. Uma delas é a modelagem das severidades. O artigo utilizado, de Verde et al. (2009), publicado pela agência de classificação de crédito Fitch, inclui títulos emitidos por governos, por exemplo, somente a partir de 2000 , e segmenta os inadimplentes de acordo com ratings da própria Fitch. Neste trabalho, somente faziam parte da amostra utilizada títulos emitidos por empresas privadas, a partir de 1986. Na maioria dos casos, não havia informação sobre a classificação de crédito dos títulos, de nenhuma das três principais agências de classificação. Portanto, apesar de a amostra utilizada para modelar as severidades ser semelhante, havia muita diferença em relação à utilizada no estudo da Fitch.
A própria amostra de carteira de crédito pode ter sido outra possível causa para a não conformidade do modelo CreditRisk+ na gestão do risco de crédito da carteira. A amostra de portfólio estudada em 1999 não necessariamente era a mesma no ano 2000. Como as amostras de portfólio eram obtidas aleatoriamente na plataforma Bloomberg, na mudança de um ano para o outro, o portfólio poderia mudar completamente, ocasionando possíveis distorções. De 2000 para 2001, por exemplo, a quantidade de eventos de inadimplência sai de 55 para 160 eventos. A variação tão grande na quantidade de defaults de um ano para o outro pode ter causa não em uma mudança de cenário da macroeconomia, mas em grandes alterações no portfólio de crédito, alterações essas que, em carteira de financiamentos de um banco, provavelmente não ocorreriam de forma tão rápida na realidade.

As variações aplicadas no modelo CreditRisk+ para análise de sensibilidade foram esclarecedoras quanto ao comportamento do modelo em distintos cenários macroeconômicos. No entanto, os fatores capazes de alterar os resultados, a frequência dos eventos de inadimplência e a severidade das perdas podem ser mais complicados do que sugere o CreditRisk+, que, por se tratar de um modelo reduzido, não se preocupa diretamente com variáveis explicativas da inadimplência ou das taxas de recuperação. Modelos estruturais podem complementar a gestão de risco de crédito de um banco, por possibilitar a incorporação de variáveis que influenciem o comportamento de parâmetros associados a crédito.

O CreditRisk + constitui um modelo cuja implementação computacional é relativamente simples, quando se considera que as perdas são independentes entre si e independentes do número de inadimplências. Para efetuar uma boa análise de risco de crédito com o CreditRisk+ é necessário haver um mercado secundário de títulos de dívidas bem desenvolvido. Sem informações de inadimplências e preços de títulos de dívida inadimplentes, a qualidade e a própria realização do estudo ficam comprometidas. No entanto, dentro do contexto de portfólios de empréstimos de bancos, o CreditRisk + torna-se uma alternativa de modelagem 
interna relevante, uma vez que uma instituição financeira pode dispor de uma base histórica de inadimplência e taxas de recuperação. Nesse caso, a implementação do CreditRisk+ torna-se viável.

Uma sugestão para estudos futuros é aplicar o modelo em uma amostra segmentada em bandas, atribuindo correlações entre os eventos de inadimplência. Outra sugestão, caso se tenha acesso a dados de carteiras de bancos, seria utilizar uma fonte de informação diferente para a severidade e para a frequência, podendo-se avaliar o modelo em uma carteira real de uma instituição financeira. No estudo conduzido, foi utilizada uma carteira hipotética bastante diversificada de títulos de dívida corporativa emitidos no mercado americano. Assim, o uso de uma carteira de crédito de operações brasileiras poderia constituir também uma importante contribuição para a análise do CreditRisk+.

Este trabalho foi realizado com o apoio da Serasa Experian (www.serasaexperian.com.br), por meio do Programa de Incentivo à Pesquisa Aplicada Serasa Experian.

\section{REFERÊNCIAS}

BABBEL, David F. Insuring Banks Against Systematic Credit Risk. The Journal of Futures Markets, v. 9, n. 6, p. 487-505, 1989.

BANCO CENTRAL DO BRASIL. Comunicado 12.746. Brasília, dez. 2004.

BOEGELEIN, L. et al. Econometric methods for sector analysis. In: CREDITRISK+ IN THE BANKING INDUSTRY. Alemanha: Springer-Verlag Berlin Heidelberg, 2004. p. 2-185.

BRINKMANN, Emile. J.; HORVITZ, Paul M. Riskbased Capital Standards and the Credit Crunch. Journal of Money, Credit and Banking, v. 27, n. 3, ago. 1995.

BRÖKER, F.; SCHWEIZER, S. Integrating rating migrations. In: CREDITRISK+ IN THE BANKING INDUSTRY. Alemanha: Springer-Verlag Berlin Heidelberg, 2004. p. 167-185.

BURGISSER, Peter. et al. Integrating Correlations. Risk Magazine, v. 12, n. 7, jul. 1999.
CAOUETTE, John B.; ALTMAN, I. E.; NARAYANAN, P. Gerenciando o risco de Crédito: o grande desafio para os mercados financeiros globais. Wiley John \& Sons, 1. Ed. 1998.

CAPUANO, C. et al. Recent Advances in Credit Risk Modeling. International Monetary Funds. Working Paper n. 09.162. Social Science Research Network, ago. 2009. Disponível em: <http://ssrn.com/abstract=1462240>. Acesso em: 10 out. 2010.

CROUHY, Michel; GALAI, Dan; MARK, Robert. A Comparative Analysis of Current Credit Risk Models. Journal of Banking \& Finance, v. 24, n. 1-2, 59-117, jan. 2000.

ÇETIN, Umut et al. Modeling Credit Risk with Partial Information. The Annuals of Applied Probability, v. 14, n. 3, p. 1167-1178, 2004.

GORDY, Michel B. A Comparative Anatomy of Credit Risk Models. Journal of Banking \& Finance, v. 24, n. 1-2, 119-149, jan. 2000.

GUNDLACH, Volker. M. Basics of CreditRisk+. In: CREDITRISK+ IN THE BANKING INDUSTRY. Alemanha: Springer-Verlag Berlin Heidelberg, 2004. p. $7-24$.

GUNDLACH, Volker. M.; LEHRBASS, Frank. B. Birth of the Model. In: CREDITRISK+ IN THE BANKING INDUSTRY. Alemanha: Springer-Verlag Berlin Heidelberg, 2004. p. 1-6.

JARROW, Robert. Default Parameter Estimation Using Market Prices. Financial Analysts Journal, v. 57, n. 5, p. 75-84, out. 2001.

MASKARA, Pankaj. Kumar; AGGARWAL, Rajesh. Credit Risk Measurement Models and their Regulatory Implications. Review of Business Research, v. 9, n. 4, p. $58-65$.

McQUOWN, John Andrew. A Comment on Market vs. Accounting-Based Measures of Default Risk. Moody's KMV Company, 1993.

MELCHIORI, Mario R. CreditRisk+ by Fast Fourier Transform. YieldCurve, ago. 2004. Disponível em: < http://ssrn.com/abstract=1122844>. Acesso em: 07 out. 2010 .

MERTON, Robert. On the pricing of corporate debt: the risk structure of interest rates. The Journal of Finance, Berkeley, v. 29, n. 2, p. 449-470, 1974. 
MOHANTY, Sunil K. Basel II: Challenges and Risks. Academy of Banking Studies Journal, v. 7, n. 2, 109130,2008 .

OJO, Marianne. Basel II and the Capital Requirements Directive: Responding to the 2008/09 Financial Crisis. Setembro 18, 2009. Disponível em: <SSRN: http://ssrn. com/abstract $=1475189>$. Acesso em 20 set. 2010.

PATEL, Kanak; PEREIRA, Ricardo. Expected Default Probabilities in Structural Models: Empirical Events. Journal of Real Estate Financial Economics, v. 34, n.1, p. 107-133, 2007.

REIB, Oliver. Dependent Sectors and an Extension to Incorporate Market Risk. In: CREDITRISK+ IN THE BANKING INDUSTRY. Alemanha: Springer-Verlag Berlin Heidelberg, 2004. p. 215-230.

SAUNDERS, Anthony. Credit Risk Measurement: New Approaches to Value-at-Risk and other Paradigms. Wiley John \& Sons, 1. Ed, p. 226, jun. 1999.

SAUNDERS, David; COSTAS, Xiouros; ZENIOS, Stavros. A. Credit Risk Optimization using Factor Models. Annals of Operation Research, v. 152, n. 1, 49 $-77,2007$.

STEVENSON, W. Estatística aplicada à administração. São Paulo: Harper \& Row do Brasil, 1981.

TASCHE, Dirk. Capital Allocation with CreditRisk+. In: CREDITRISK+ IN THE BANKING INDUSTRY. Alemanha: Springer-Verlag Berlin Heidelberg, 2004. p. 25-44.

THOMAS, Lyn C. Consumer Credit Models: Pricing, Profit and Portfolios. United States of America: Oxford University Press, abr. 2009. 400 p.

THOMAS, L. C.; OLIVER, R. W.; HAUD, D. J. A survey of the issues in consumer credit modelling research. Journal of the Operational Research Society, v. 56, 1006-1015, mai. 2005.

VERDE, Mariarosa. et al. Default Surge, Recoveries Sink in 2009: Understanding the Fundamental and Cyclical Drivers of Corporate Recovery Rates. Fitch Ratings, jul. 2009.

WILDE, T. CreditRisk+: A CreditRisk Management Framework, Credit Suisse First Boston, CreditRisk+ Technical document (1997) 